\section{EARTHWORMS FROM NORTHERN INDIAN STATES WITH OCNERODRILUS OCCIDENTALIS EISEN, 1878 AS A NEW REPORT FROM PUNJAB}

\author{
Neena Dhiman ${ }^{1,2}$ and S.K. Battish ${ }^{1}$ \\ ${ }^{1}$ Department of Zoology and Fisheries, Punjab Agricultural \\ University, Ludhiana, Punjab 141004, India \\ Email: ${ }^{2}$ neena_dhiman@hotmail.com
}

The published work on terrestrial oligochaetes of India includes short publications as well as work on comprehensive fauna. Michaelsen (1909) published a consolidated account on taxonomic studies of Indian oligochaetes including keys for identification of and description of all known species in India and adjacent countries until then. Stephenson (1923) documented the earlier work on earthworms in the Fauna of British India volumes. Gates' (1972) work culminated in the publication of a monograph, including the description of species from northeastern India and Andaman and Nicobar Islands, while Julka (1988) further substantiated work on Oligochaeta. This note lists thirty species of earthworms including twenty from Punjab and the Union Territory of Chandigarh and reports Ocnerodrilus occidentalis Eisen (1878) as new record from Punjab.

Material and Methods: Earthworms were collected regularly from various ecological niches such as litter, kitchen drainage, manure heaps, different types of soils, margins of freshwater bodies, pastures, grasslands, forests, cultivated fields etc. spread over the northern Indian states including Punjab, Haryana, Himachal Pradesh, Uttaranchal, Delhi and adjoining areas. These areas were explored for a period of more than three years, from July 1999 to August 2003, except in the months of December and January due to non availability of worms during extreme cold conditions in the upper soil profile. Live worms were brought to laboratory for identification. For diagnostic taxonomic characters, they were dissected after narcotizing in $30 \%$ ethyl alcohol, fixing in $10-15 \%$ formalin for 24 hours and finally preserving in $10-12 \%$ formalin. The anatomical details were examined under dissecting binocular microscope.

Results and Conclusions: The species so identified belong to six families namely Eudrilidae, Lumbricidae, Moniligastridae, Megascolecidae, Ocnerodrilinae and Octochaetidae and are presented in Table 1 along with information regarding important taxonomic features for each species. The habitat of their occurrence and month of collection for each species is also given. The most widely distributed species found in Punjab is Metaphire posthuma followed by Lampito mauritii and Amynthas morrisi. However, Eudrilus eugeniae and Eisenia fetida are best for vermiculture. Potentialities in future also exist to exploit Perionyx excavatus and Allolobophora parva for vermiculture. Ocnerodrilus occidentalis is reported for the first time from Punjab. Perionyx barotensis is strictly available in district Barot of Himachal Pradesh and so far is not recovered elsewhere.

\section{REFERENCES}

Gates, G.E. (1972). Burmese earthworms An introduction to the systematics and biology of megadrile oligochaetes with special reference to Southeast Asia. Transactions of the American Philosophical Society NS 62: 1-326.

Julka, J.M. (1988). The Fauna of India and the adjacent countries Megadrile oligochaeta (Earthworms) Haplotaxida: Lumbricina: Megascolecoidea: Octochaetidae. Zoological Survey of India, Calcutta, 400pp+xiv.

Michaelsen, W. (1909). The Oligochaeta of India, Nepal, Ceylon, Burma and the Andaman Islands. Memories of Indian Museum I.

Stephenson, J. (1923). Oligochaeta. In: The Fauna of British India. Francis and Taylor, London.

Stephenson, J. (1930). The Oligochaeta. Clarendon Press, Oxford, 978pp.

\section{ACKNOWLEDGEMENTS}

The authors are thankful to Dr. J.M. Julka, Consultant, Earthworm Identification and Vermiculture, Zoological Survey of India, Solan for his timeless help in identification and confirmation of species and for providing valuable suggestions during the studies.

Table 1. Earthworms from northern Indian states

\begin{tabular}{|c|c|c|c|c|c|c|c|c|}
\hline Species & Location & $\begin{array}{l}\text { Month of } \\
\text { collection }\end{array}$ & Habitat & $\begin{array}{l}\text { Avg length; } \\
\text { Diameter }(\mathrm{cm})\end{array}$ & $\begin{array}{l}\text { Total segments / } \\
\text { Ciltellar segments / } \\
\text { Type of clitellum }\end{array}$ & $\begin{array}{l}\text { Prosto- } \\
\text { mium }\end{array}$ & $\begin{array}{l}\text { Location of } \\
\text { prostate } \\
\text { glands }\end{array}$ & $\begin{array}{l}\text { Spermathecae } \\
\text { number I } \\
\text { Location }\end{array}$ \\
\hline $\begin{array}{l}\text { Family: Eudrilidae } \\
\text { Eudrilus eugeniae } \\
\text { Kinberg, } 1867\end{array}$ & Chandigarh & May, 2001 & $\begin{array}{l}\text { Shaded grasslands, } \\
\text { mud }\end{array}$ & $12-14 ; 0.48$ & 200; XII-XVII; A & Epi & $\begin{array}{l}\text { XVI-XXVI; } \\
\text { As euprostates }\end{array}$ & 1 pair; XIV \\
\hline $\begin{array}{l}\text { Family: Lumbricidae } \\
\text { Allolobophora } \\
\text { caliginosa caliginosa } \\
\text { Beddard, } 1895\end{array}$ & $\begin{array}{l}\text { Kullu, Manali, } \\
\text { Solan (H.P.) }\end{array}$ & May, 2001 & $\begin{array}{l}\text { Earth around potted } \\
\text { plants, gardens, } \\
\text { fields, compost }\end{array}$ & $6-16 ; 0.45$ & $\begin{array}{l}\text { 100-245; XXVI-XVII or } \\
\text { XXVIII-XXXV; S }\end{array}$ & Epi & XXXII-XXXIV & $2 ; 9 / 10,10 / 11$ \\
\hline $\begin{array}{l}\text { Allolobophora parva } \\
\text { Eisen, } 1874\end{array}$ & $\begin{array}{l}\text { Roopnagar, } \\
\text { Nawanshehar, } \\
\text { Kajli, Jodhan, } \\
\text { Marhana, Thaka, } \\
\text { Aujla, Narangwal, } \\
\text { Dhaliwal (Punjab) }\end{array}$ & $\begin{array}{l}\text { April, } 2003 \text { - } \\
\text { August, } 2003\end{array}$ & $\begin{array}{l}\text { Waste effluents of } \\
\text { habitations, under logs } \\
\text { gardens, fields, } \\
\text { humus, manure }\end{array}$ & $2.5-4 ; 0.14$ & 85-124; XXIV-XXX; S & Epi & $X V-X V I$ & Lacking \\
\hline $\begin{array}{l}\text { Dendrodrilus rubidus } \\
\text { Savigny, } 1826\end{array}$ & Manali (H.P.) & July, 2002 & $\begin{array}{l}\text { Litter, compost, } \\
\text { fields, meadows }\end{array}$ & $5-6 ; 0.35$ & $\begin{array}{l}\text { 74-110; XXV or } \\
\text { XXVI-XXXI or XXXII; S }\end{array}$ & Epi & Unidentifiable & $2 ; 9,10$ \\
\hline
\end{tabular}


Table 1. Earthworms from northern Indian states

\begin{tabular}{|c|c|c|c|c|c|c|c|c|}
\hline Species & Location & $\begin{array}{l}\text { Month of } \\
\text { collection }\end{array}$ & Habitat & $\begin{array}{l}\text { Avg length; } \\
\text { Diameter }(\mathrm{cm})\end{array}$ & $\begin{array}{l}\text { Total segments / } \\
\text { Ciltellar segments / } \\
\text { Type of clitellum }\end{array}$ & $\begin{array}{l}\text { Prosto- } \\
\text { mium }\end{array}$ & $\begin{array}{l}\text { Location of } \\
\text { prostate } \\
\text { glands }\end{array}$ & $\begin{array}{l}\text { Spermathecae } \\
\text { number / } \\
\text { Location }\end{array}$ \\
\hline $\begin{array}{l}\text { Eisenia fetida } \\
\text { Savigny, } 1826\end{array}$ & Chandigarh & May, 2001 & $\begin{array}{l}\text { In debris, mess, } \\
\text { gardens, sewage, } \\
\text { streams, meadows }\end{array}$ & $12-13.5 ; 0.46$ & $\begin{array}{l}90-125 ; \mathrm{XXIV,XXV,XXVI} \\
\text { to XXXIV; S }\end{array}$ & Epi & Absent & $2 ; 9,10$ \\
\hline $\begin{array}{l}\text { Octolasion tyrtaeum } \\
\text { Savigny, } 1826\end{array}$ & $\begin{array}{l}\text { Banjar district } \\
\text { (Kullu) }\end{array}$ & $\begin{array}{l}\text { May, } 2000 \\
\& \text { April, } 2001\end{array}$ & $\begin{array}{l}\text { Under stones, logs, } \\
\text { compost, litter, mud }\end{array}$ & $3-12 ; 0.30$ & 75-120; XXX-XXXV; S & Epi & XIV-XV & $2 ; 10,11$ \\
\hline \multicolumn{9}{|c|}{ Family: Megascolecidae } \\
\hline $\begin{array}{l}\text { Amynthas alexandri } \\
\text { Beddard, } 1900\end{array}$ & $\begin{array}{l}\text { Ambala, } \\
\text { Roopnagar } \\
\text { (Punjab) }\end{array}$ & $\begin{array}{l}\text { April, } 1999 \\
\text { \& July, } 2001\end{array}$ & $\begin{array}{l}\text { Gardens, lawns, } \\
\text { fields, jungles }\end{array}$ & $\begin{array}{l}11-12.5 \\
0.46-0.49\end{array}$ & 90-140; XIV-XVI; A & $\begin{array}{l}\text { Rudi- } \\
\text { mentary }\end{array}$ & XVI-XXII & $4 ; 6 / 7-9 / 10$ \\
\hline $\begin{array}{l}\text { Amynthas corticis } \\
\text { Kinberg, } 1867\end{array}$ & $\begin{array}{l}\text { Nangal, } \\
\text { Roopnagar } \\
\text { (Punjab) }\end{array}$ & $\begin{array}{l}\text { March, } 2003 \\
\text { to May, } 2003\end{array}$ & $\begin{array}{l}\text { Gardens, fields, } \\
\text { forests }\end{array}$ & $8-9 ; 0.45-0.47$ & 90-100; XIV-XVI; A & Epi & Absent & $4 ; 5 / 6-8 / 9$ \\
\hline $\begin{array}{l}\text { Amynthas gracilis } \\
\text { Kinberg, } 1867\end{array}$ & $\begin{array}{l}\text { Ambala, } \\
\text { Amritsar (Punjab) }\end{array}$ & April, 2002 & $\begin{array}{l}\text { Gardens, green } \\
\text { houses, drainages }\end{array}$ & $\begin{array}{l}9-10 \\
0.35-0.43\end{array}$ & 60-100; XIV-XVII; A & Epi & XVI-XXIV & $3 ; 5 / 6-7 / 8$ \\
\hline $\begin{array}{l}\text { Amynthas morrisi } \\
\text { Beddard, } 1892\end{array}$ & $\begin{array}{l}\text { Ludhiana } \\
\text { open areas }\end{array}$ & July, 2003 & Garden soils, lawns, & $\begin{array}{l}9.5-11.5 \\
0.45-0.50\end{array}$ & 90-110; XIV-XVI; A & Epi & XVII-XXII & $2 ; 5 / 6,6 / 7$ \\
\hline $\begin{array}{l}\text { Lampito mauritii } \\
\text { Kinberg, } 1866\end{array}$ & $\begin{array}{l}\text { Ludhiana, } \\
\text { Doraha, Sirhind, } \\
\text { Kapurthala, } \\
\text { Hoshiarpur, Jalan }\end{array}$ & $\begin{array}{l}\text { June, } 2001 \text { to } \\
\text { August, } 2003 \\
\text { ndhar (Punjab) }\end{array}$ & $\begin{array}{l}\text { Gardens, manure } \\
\text { heaps, fields }\end{array}$ & $\begin{array}{l}9-13.5 \\
0.29-0.32\end{array}$ & 168-195; XIV-XVII; A & Pro & XVIII & $3 ; 6 / 7-8 / 9$ \\
\hline $\begin{array}{l}\text { Metaphire houlleti } \\
\text { Perrier, } 1872\end{array}$ & $\begin{array}{l}\text { Himachal } \\
\text { Pradesh }\end{array}$ & March, 2001 & $\begin{array}{l}\text { Gardens, lawns, } \\
\text { Jungles }\end{array}$ & $\begin{array}{l}9.2-20 \\
0.50-0.52\end{array}$ & 9.2-120; XIV-XVI; A & Epi & XVI-XXI & $3 ; 6 / 7-8 / 9$ \\
\hline $\begin{array}{l}\text { Metaphire posthuma } \\
\text { Vaillant, } 1868\end{array}$ & $\begin{array}{l}\text { Ludhiana, } \\
\text { Gurdaspur, } \\
\text { Phillaur, Faridkot, } \\
\text { Jalandhar, Kapur }\end{array}$ & $\begin{array}{l}\text { May, } 2001 \text { to } \\
\text { August, } 2003 \\
\text { t, } \\
\text { rthala (Punjab), }\end{array}$ & $\begin{array}{l}\text { Lawns, gardens, mud } \\
\text {, Anandpur sahib, Gar }\end{array}$ & $\begin{array}{l}0.80-1.30 \\
0.45-0.65 \\
\text { rshankar forests }\end{array}$ & $\begin{array}{l}90-135 ; \text { XIII 1/2-XVI 1/2 } \\
\text { or XVII; A } \\
\text {,, Delhi, Ambala, Panip }\end{array}$ & Epi & $X V-X X I$ & $4 ; 5 / 6-8 / 9$ \\
\hline $\begin{array}{l}\text { Polypheretima elongata } \\
\text { Perrier, } 1872\end{array}$ & $\begin{array}{l}\text { Ludhiana, Sirhind } \\
\text { Roopnagar } \\
\text { (Punjab) }\end{array}$ & $\begin{array}{l}\text { d, April to June } \\
\text { 2000; May to } \\
\text { June, } 2001\end{array}$ & $\begin{array}{l}\text { e,Dairy farm yard, } \\
\text { gardens, compost }\end{array}$ & $\begin{array}{l}15-21 \\
0.44-0.47\end{array}$ & 150-260; XIV-XVI; A & $\begin{array}{l}\text { Rudi- } \\
\text { mentary }\end{array}$ & XVI-XXI & $2 ; 5 / 6,6 / 7$ \\
\hline $\begin{array}{l}\text { Perionyx bainii } \\
\text { Stephenson } 1915\end{array}$ & Kullu & June, 2001 & $\begin{array}{l}\text { Soils with rich organic } \\
\text { matter and leaf litter }\end{array}$ & $\begin{array}{c}\text { c } 0.25-0.45 \\
0.23-0.25\end{array}$ & 90-95; XIII-XVII; A & Epi & XVIII & $2 ; 7 / 8,8 / 9$ \\
\hline $\begin{array}{l}\text { Perionyx barotensis } \\
\text { Jhulka, } 1993\end{array}$ & Baroti (H.P.) & May, 1999 & $\begin{array}{l}\text { Soils with rich organic } \\
\text { matter and leaf litter }\end{array}$ & $\begin{array}{l}\text { c } 7-9.5 \\
0.23-0.25\end{array}$ & 105-130; XIII-XVII; A & Epi & $X X I-X X I I I$ & $2 ; 8,9$ \\
\hline $\begin{array}{l}\text { Perionyx excavatus } \\
\text { Perrier, } 1872\end{array}$ & Palampur (H.P.) & $\begin{array}{l}\text { May, } 2002 \text { to } \\
\text { April, } 2003\end{array}$ & $\begin{array}{l}\text { Debris, under stones, } \\
\text { sandy soils }\end{array}$ & 0.30-1.80; & $\begin{array}{l}120-155 ; \text { XIII-XVII; A } \\
0.42-0.45\end{array}$ & Epi & XVIII & $2 ; 7 / 8,8 / 9$ \\
\hline $\begin{array}{l}\text { Perionyx sansibaricus } \\
\text { Michaelsen, } 1891\end{array}$ & $\begin{array}{l}\text { Gurdaspur, } \\
\text { Pathankot } \\
\text { (Punjab) }\end{array}$ & $\begin{array}{l}\text { March, } 2001 \\
\text { \& April, } 2001\end{array}$ & $\begin{array}{l}\text { Soil with high organic } \\
\text { matter and moisture }\end{array}$ & $\begin{array}{l}3.2-6.5 \\
0.29-0.30\end{array}$ & 80-105; XIII-XVII; A & Epi & XVII-XIX & $3 ; 7,8,9$ \\
\hline $\begin{array}{l}\text { Perionyx simlaensis } \\
\text { Michaelsen, } 1907\end{array}$ & $\begin{array}{l}\text { Dholvahe (H.P.), } \\
\text { Hoshiarpur (Punja }\end{array}$ & $\begin{array}{l}\text { April, } 2002 \\
\text { ab) }\end{array}$ & $\begin{array}{l}\text { Soils rich in organic } \\
\text { matter }\end{array}$ & $\begin{array}{l}8.5-10 \\
0.42-0.45\end{array}$ & 120-135; XIII-XVII; A & Epi & XVII & $2 ; 7 / 8,8 / 9$ \\
\hline $\begin{array}{l}\text { Family: Ocnerodrilina } \\
\text { Gordiodrilus elegans } \\
\text { peguanus Beddard, } \\
1892\end{array}$ & $\begin{array}{l}\text { Roopnagar, } \\
\text { Mirzapur stream } \\
\text { bank }\end{array}$ & $\begin{array}{l}\text { October, } 2001 \\
\& \text { November, } \\
2002\end{array}$ & Forest, lake shores & $2.6-4.7 ; 0.15$ & $\begin{array}{l}\text { 80-95; XIII-XVIII, } \\
\text { XIX, XX; A }\end{array}$ & Epi & XVII-XVIII & $2 ; 7 / 8,8 / 9$ \\
\hline $\begin{array}{l}\text { Ocnerodrilus } \\
\text { occidentalis Eisen, } 1878\end{array}$ & $\begin{array}{l}\text { Ludhiana } \\
8 \text { (Punjab) }\end{array}$ & $\begin{array}{l}\text { May, } 2002 \text { to } \\
\text { July, } 2002\end{array}$ & Soils of potted plants & $1.5-3 ; 0.11$ & $\begin{array}{l}65-70 ; \mathrm{XIII} \text { or XIV-XIX } \\
\text { or XX; A }\end{array}$ & $\begin{array}{l}\text { Unidenti- } \\
\text { fiable }\end{array}$ & XVII-XIX & Absent \\
\hline $\begin{array}{l}\text { Family: Octochaetidae } \\
\text { Dichogaster bolaui } \\
\text { Michaelsen, } 1900\end{array}$ & Roopnagar & February, 2000 & $\begin{array}{l}0 \text { Soils rich in organic } \\
\text { matter }\end{array}$ & $2-4 ; 0.13-0.15$ & $\begin{array}{l}\text { 75-95; XIII or XIV- } \\
\text { XVIII, XIX, XX; S }\end{array}$ & Pro-epi & XVII, XIX & $2 ; 7 / 8,8 / 9$ \\
\hline $\begin{array}{l}\text { Eutyphoeus ibrahimi } \\
\text { Stephenson, } 1914\end{array}$ & Kapurthala & July, 2000 & $\begin{array}{l}\text { Fields, gardens, } \\
\text { margins of aquatic bc }\end{array}$ & $\begin{array}{l}7 ; 0.23-0.25 \\
\text { odies }\end{array}$ & 185 ; indefinite clitellum & n Tany & Not defined & $1 ; 7 / 8$ \\
\hline $\begin{array}{l}\text { Eutyphoeus } \\
\text { incommodus Beddard, } \\
1901\end{array}$ & $\begin{array}{l}\text { Nandpur, } \\
\text { Sanehawal, } \\
\text { Hoshiarpur } \\
\text { (Punjab), Ambala }\end{array}$ & $\begin{array}{l}\text { April, } 2002 \text { \& } \\
\text { May, } 2002\end{array}$ & $\begin{array}{l}\text { Soils rich in organic } \\
\text { matter and moisture }\end{array}$ & $9-11 ; 0.40$ & $\begin{array}{l}\text { 120-150; XIII-XVII or } \\
\text { XVIII; A }\end{array}$ & Pro-epi & $\mathrm{XVII}$ & $1 ; 7 / 8$ \\
\hline $\begin{array}{l}\text { Eutyphoeus waltoni } \\
\text { Michaelsen, } 1907\end{array}$ & $\begin{array}{l}\text { Chohal, } \\
\text { Hoshiarpur } \\
\text { (Punjab), New De }\end{array}$ & $\begin{array}{l}\text { April, } 2003 \text { \& } \\
\text { July, } 1999 \\
\text { elhi }\end{array}$ & $\begin{array}{l}\text { In dense vegetation } \\
\text { deep in earth }\end{array}$ & $\begin{array}{l}9-20 \\
0.45-0.50\end{array}$ & $\begin{array}{l}190-210 ; 1 / 2 \text { XIII- } \\
\text { XVII; A }\end{array}$ & Tany & XIV-XVII & $1 ; 7 / 8$ \\
\hline
\end{tabular}




\begin{tabular}{|c|c|c|c|c|c|c|c|c|}
\hline Species & Location & $\begin{array}{l}\text { Month of } \\
\text { collection }\end{array}$ & Habitat & $\begin{array}{l}\text { Avg length; } \\
\text { Diameter (cm) }\end{array}$ & $\begin{array}{l}\text { Total segments / } \\
\text { Ciltellar segments / } \\
\text { Type of clitellum }\end{array}$ & $\begin{array}{l}\text { Prosto- } \\
\text { mium }\end{array}$ & $\begin{array}{l}\text { Location of } \\
\text { prostate } \\
\text { glands }\end{array}$ & $\begin{array}{l}\text { Spermathecae } \\
\text { number / } \\
\text { Location }\end{array}$ \\
\hline $\begin{array}{l}\text { Lennogaster } \\
\text { chittagongensis } \\
\text { Stephenson, } 1917\end{array}$ & $\begin{array}{l}\text { Nathwai, } \\
\text { Sukhna WLS }\end{array}$ & $\begin{array}{l}\text { September, } \\
2002\end{array}$ & Soil rich in moisture & $2.4-7 ; 0.15$ & 120; XIII-XVII; A & Epi & $\mathrm{XVII}$ & $1 ; 8$ \\
\hline $\begin{array}{l}\text { Lennogaster pusillus } \\
\text { Stephenson, } 1920\end{array}$ & $\begin{array}{l}\text { Kansal forest, } \\
\text { Chandigarh (U.T. }\end{array}$ & August, 2000 & $\begin{array}{l}\text { Soils rich in organic } \\
\text { matter, leaf litter and }\end{array}$ & $\begin{array}{l}2.5-4.5 ; 0.13 \\
\text { humus }\end{array}$ & 100-125; XIII-XVII; A & Pro-epi & $\mathrm{XVII}$ & $1 ; 8$ \\
\hline $\begin{array}{l}\text { Octochaetona beatrix } \\
\text { Beddard, } 1902\end{array}$ & Hoshiarpur & March, 2001 & $\begin{array}{l}\text { Earth of potted } \\
\text { plants }\end{array}$ & $7-8 ; 0.35$ & $\begin{array}{l}\text { 170-180; XIII-XVII, } \\
\text { XVIII; A }\end{array}$ & Epi & XVII \& XIX & $2 ; 8,9$ \\
\hline $\begin{array}{l}\text { Ramiella } \\
\text { bishamberensis } \\
\text { Stephenson, } 1914\end{array}$ & $\begin{array}{l}\text { Forest near } \\
\text { Sukhna lake, } \\
\text { Chandigarh (U.T. }\end{array}$ & $\begin{array}{l}\text { August, } 2000 \\
\text { Г.) }\end{array}$ & Moist neutral soils & $3.5 ; 0.1$ & 85; XIV-XVI; A & Epi & XVII-XIX & $2 ; 8,9$ \\
\hline $\begin{array}{l}\text { Family: Moniligastri } \\
\text { Drawida japonica } \\
\text { Michaelson, } 1917\end{array}$ & $\begin{array}{l}\text { dae } \\
\text { Solan }\end{array}$ & June, 2003 & $\begin{array}{l}\text { Soils rich in organic } \\
\text { matter }\end{array}$ & $3-6 ; 0.26$ & $\begin{array}{l}\text { 130-142; } \\
\text { inconspicuous clitellum }\end{array}$ & Pro & $x$ & $1 ; 7 / 8$ \\
\hline
\end{tabular}

Tany - Tanylobous; Pro - Prolobous; Epi - Epilobous; S - Saddle; A - Annular

NOTE

ZOOS' PRINT JOURNAL 21(1): 2137-2139

\section{ICHTHYOFAUNA FROM FOUR DISTRICTS OF MARATHWADA REGION, MAHARASHTRA, INDIA}

\section{C.J. Hiware}

Reader, Department of Zoology, Dr. Bababsaheb Ambedkar Marathwada University, Aurangabad, Maharashtra 431004, India Email: drhiware@rediffmail.com

The Marathwada region of Maharashtra is one of the richest in aquatic resources that includes tributaries of river Godavari, Purna, Painganga, Manjara and Dudhana apart from reservoirs and lakes. In the field of ichthyology valuable contributions have been made by Ahirrao and Mane (2000) who studied ichthyofauna from Parbhani district of Maharashtra state and Sakhare (2001) who studied ichthyofauna of Jawalgaon Reservior in Solapur district in Maharashtra state. The present work was mainly undertaken to investigate the fish diversity from this region and it is first effort in this direction.

The survey work was mainly undertaken in four major districts, namely, Aurangabad, Nanded, Parbhani and Osmanabad of Marathwada region. From these districts some of the places having small and big water resources with fishery potential were selected for collection of ichthyofauna. Some of the important places under study include:

\section{Aurangabad district:}

Collection sites: Aurangabad, Paithan, Werul, Ajantha, Sillod. Water resources: Godavari river, Kham river, Nathsagar dam, Purna river, Salim Ali lake, Harsool lake, Tisgaon pond, Khajan pond, etc.

\section{Parbhani district:}

Collection sites: Parbhani, Yeldari, Purna, Jintur etc.

Water resources: Purna river, Kapra river, Masoli lakes, Yeldari dam, Jam pond, Rahati pond, etc.

\section{Nanded district:}

Collection sites: Nanded, Vishnupuri, Kandhar, Kinwat, Mahur Water resources: Godavari river, Manar river, Painganga river, Vishnupuri dam, Jagatunga Samudra reservoir, Dhamdari pond.

\section{Osmanabad district:}

Collection sites: Osmanabad, Tuljapur, Naldurg, Kallam, Bhum Water resources: Terna river, Manjara river, Sina river, Bori dam, Tugao pond, etc.

Fishes were collected during regular monthly between May 2003 and April 2004 from the local fish markets and fishing spots of different places. The local fishermen fish using different types of gill nets, cast nets, Maccharjali, disco nets operated through indigenously designed rafts and Ratnagiri type boats. After systematic identification the specimens were preserved in $4 \%$ formalin and deposited in the Zoology Department Museum, Dr. Babasaheb Ambedkar Marathwada University Aurangabad after providing appropriate registration numbers and other details. The identification of the fishes were carried out with the help of standard literature (Day, 1878; Jayram, 1981; Qureshi \& Qureshi, 1983; Datta \& Srivastava, 1988; Talwar \& Jhingran, 1991).

Sixty-six fish species belonging to 33 genera, 16 families and eight orders were recorded from the freshwaters of four districts of Marathwada region in Maharashtra state (Table 1). The ichthyofauna consisted of carps, catfishes and trash fishes. The carps dominated overall over other groups throughout the year. The group also supported capture as well as culture fishery

Manuscript 1210: Received 28 May 2004: Revised received 16 July 2004: Finally accepted 20 September 2005 ; Date e organisation; www.zoosprint.org 